\title{
"Evaluation of the Efficacy of Azadirachta Indica (Neem) Extract Gel as a Local Drug Delivery in the Treatment of Patients with Chronic Periodontitis." A Double Blind Randomised Clinical Trial.
}

\author{
Dr. Verdine Virginia Antony ${ }^{1}$, Dr. Deepak Prasad ${ }^{2}$, Dr. Rahamath ulla khan ${ }^{3}$ \\ ${ }^{1}$ (Department of Periodontics, Sirte University, Libya.) \\ ${ }^{2}$ (Principal, Farooqia Dental college, Mysore.) \\ 3 (Department of Orthodontics, Sirte University, Libya.)
}

\begin{abstract}
Introduction: Periodontal diseases comprise of a group of inflammatory conditions of the supporting tissues of the teeth that are initiated by microorganism. The removal or inhibition of subgingival plaque and maintenance is dependent on continued plaque control for which adjunctive local antimicrobial therapy has shown promising results. In the present study, we explored the efficacy of Azadirachta indica (Neem) extract gel as a local drug delivery in the treatment of patients with chronic periodontitis and evaluated the clinical as well as the microbiological benefits when used as an adjunct to scaling and root planning.

Method: 20 patients with chronic periodontitis in the age group of 30-55 years, with pocket depth of $\geq$ $5 \mathrm{~mm}$ with radiographic evidence of bone loss participated in the study. Following scaling and root planing the neem extract gel and placebo gel were placed at the experimental and control sites respectively. The clinical parameters were recorded at baseline, 1 month, 3 months and 6 months while the microbiological parameters were recorded at baseline, 7 days, 15 days and 30 days respectively.

Results: The results showed improvements in the clinical parameters both at the control and the experimental sites. However, the experimental sites showed better results compared to the control site. The microbiological analysis proved a relative reduction of the periodontal pathogens at both the experimental as well as the control site with the control site showing a significantly higher reduction.

Conclusion: Neem extract gel produced more improvements in clinical as well as microbiological parameters than the control. The approach of local delivery of a natural extract with no known side effects has proved promising results.
\end{abstract}

Keywords: Azadirachta indica (Neem), Clinical parameters, microbiological parameters, Therapeutic use.

\section{INTRODUCTION}

Periodontal disease is an infectious, inflammatory disease and its initiation and progression is significantly associated with overgrowth of certain pathogenic bacteria, liberation of bacterial toxins and inflammatory response of the host. ${ }^{1}$ Conventional periodontal treatment aims at reducing or completely eliminating the pathogenic microbes from the periodontal pockets and altering the subgingival microbiota that was associated with progressive destruction. But conventional mechanotherapy has certain limitations. ${ }^{2}$ Therefore, antimicrobial agents may be used adjunctively to scaling and root planing in the management of periodontitis. Various investigators have advocated the use of antimicrobials, initially systemically and more recently locally for the management of chronic periodontitis. ${ }^{3}$ Local route of drug delivery can accomplish 100 fold higher therapeutic doses of the agents in sub gingival sites, than those possible by systemic therapy. In recent years, there have been reports suggesting that mechanical debridement with locally delivered antimicrobials used adjunctively, is highly effective in the management of periodontal disease. ${ }^{4}$

Neem has been used in India and South Asia for thousands of years as the preferred tool for maintaining healthy gums and teeth. Brushing with neem twigs and chewing neem leaves and seeds after a meal has been the traditional dental care practice. With available modern preparations many people are now using commercial products that contain the same basic neem components. The antibacterial activity of neem has been evaluated and known from ancient times. ${ }^{5,6}$ Neem has been considered to have various therapeutic activities such as astringent, antiseptic, insecticidal, antiulcer and for cleaning the teeth in pyorrhoea and other dental diseases. Other than this, leaf extract of the neem showed superior antiviral and antihyperglycemic activity in vitro and in vivo on animals. ${ }^{7}$ Leaves of the neem have been used in the treatment of gingivitis and periodontitis. Neem has also showed better efficacy in the treatment of oral infections and plaque growth inhibition in treating 
periodontal disorders. ${ }^{8}$ Neem has also shown good in vitro, broad range antibacterial activity. ${ }^{9}$ It removes toxins from the body, purifies the blood and neutralizes damaging free radicals. So, locally it may also have an effect in enhancing healing. Neem extract contains (i) Azadiractin - the active principle, (ii) glycosides -antimicrobial, (iii) sterols (iv) luminols-anti-inflammatory and (v) flavenoids. ${ }^{9}$

The above ingredients and their properties along with the easy availability of neem, with no known adverse reactions and being cost effective, has been selected for this study as a local drug delivery in the gel form, to be placed in the periodontal pocket and to evaluate improvement in the clinical parameters and reduction of microbial flora. The purpose of this study was to determine the efficacy of Azadirachta indica (neem) extract gel $(20 \mathrm{mg} / \mathrm{g})$ as a local drug delivery system along with scaling and root planing in the management of moderate to severe chronic periodontitis.

\section{METHODOLOGY}

A total of 20 patients were selected including both males and females in the age group of $30-55$ years were considered

Inclusion criteria: Patients diagnosed to have chronic periodontitis with probing pocket depth $\geq 5 \mathrm{~mm}$ with radiographic evidence of bone loss, free from any systemic disease and who had not undergone any form of non-surgical or surgical periodontal therapy in the last 6 months.

Exclusion criteria: Pregnant ladies or lactating mothers, patients having systemic diseases, smokers, patients who had received periodontal treatment within 6 months of enrolment into the study, Patients who had received any topical or systemic antibiotic treatment for any purpose in the past 6 months including the use of mouth wash or currently on systemic antibiotics.

\section{II.1 Clinical trial design}

The nature and design of the clinical trial was explained to the patients and consent was obtained .Oral hygiene instructions for supragingival plaque control were given.

The study used a split-mouth design wherein two sites in the contralateral quadrants which required periodontal treatment with probing pocket depths of at least $\geq 5 \mathrm{~mm}$ at baseline were chosen and randomly assigned for the double blind study. A total of 40 sites from 20 patients were selected for this study.

Impressions of the upper and lower arches were made using alginate impression material and casts were poured in dental stone and Acrylic stents The pocket depth was measured using a constant pressure probe (Brock probe $\left.{ }^{\mathrm{TM}}\right) .{ }^{10}$

The selected sites were grouped as:

Group A: These sites received scaling and root planing along with Gel 1. [Figure: 2.a]

Group B: These sites received scaling and root planing along with Gel 2. [Figure: 2.b]

After the placement of the Gels 1 and 2. The treated areas were given periodontal pack . The clinical parameters of the selected target sites were undertaken before placement of the gel at baseline [Figure: 1.a, 1.b] , 1month [Figure: 3.a, 3.b] , 3months and 6 months follow up. [Figure: 4.a, 4.b] At all recall visits only supra gingival scaling was done.

The following clinical parameters were used to assess the periodontal status: Plaque Index (Silness and Loe, 1964) ${ }^{11}$, Gingival index (Loe and Silness, 1963) ${ }^{11}$,Probing Pocket Depth (PPD) ${ }^{12}$ and Clinical attachment level $(\mathrm{CAL})^{12}$

\section{2 Microbial procedure}

On day 0 , prior to the placement of the formulations the plaque sample was taken for bacteriological analysis from the selected sites. The supragingival plaque was removed to prevent contamination of the flora, and then the area was isolated and subgingival plaque was collected using a sterilized Gracey curette .The samples were transferred to a bottle containing thioglycollate transport media. This procedure was again repeated on day 7 , day 15 and day 30 .

\section{3 Microbial analysis}

The microbial analysis consisted of a gram's stain were in morphotypes of organisms were conducted. The bacterial culturing was done using Agar supplemented with defibrinated and hemolysed horse blood, anaerobic sachets and jars. Organisms were provisionally identified on their colony characteristics and the colony count taken. The colony count was expressed in numbers/sample. Smears were prepared from the colonies. Gram's stained and the colony morphology noted to detect the specific organisms namely Porphyromonas gingivalis, Prevotella intermedia, Capnocytophaga, Fusobacterium nucleatum.

\section{4 Formulation of gels}

- Placebo gel- Carbopol 934P gel and Gellan

- Neem gel containing $500 \mathrm{mg}$ of Neem extract (5\% \& $20 \% \mathrm{w} / \mathrm{w})$ in required amount of distilled water, 1 gm of Carbopol 934P , Triethanolamine, $75 \mathrm{mg}$ of methyl paraben and $25 \mathrm{mg}$ propyl paraben Sterility 
Testing of Formulations ${ }^{13}$ was performed using Nutrient broth media for aerobic bacteria and Fluid thioglycollate media for anaerobic bacteria.

\section{RESULTS}

The Plaque Index score [TABLE:1] in both the experimental and control site showed a statistically highly significant $(\mathrm{p}<0.000)$ improvement over a duration of six months. The mean change in Plaque index at the experimental sites was $0.9000 \pm 0.2487$ which was greater than that observed at the control site $(1.3500 \pm$ 0.4617). This indicated a better performance by the experimental site compared to the control, with the Neem group showing better results. ${ }^{14}$ A statistically highly significant $(p<0.000)$ reduction in Gingival Index [TABLE:2] was observed at both experimental and control sites over six months. A mean change of $0.7375 \pm$ 0.2361 at the experimental site indicated a higher reduction in the Gingival Index compared to the control site which showed a mean change of $1.3250 \pm 0.2308$ over six months.

A statistically highly significant reduction $(\mathrm{p}<0.000)$ in the probing pocket depth as well as the clinical attachment level in both the test as well as the control groups from baseline to 6 months was noted and were maintained till the end of the study but with Group A (Test site) showing a greater significance when compared to Group B (Control Site) [TABLE:3a,3b].

In the microbiological analysis a mean decrease in the gram positive cocci, gram negative cocci, gram positive bacilli and gram negative bacilli was observed in both the groups when compared from day 0[Figure: 5]. to day 30 [Figure: 6]. Comparison between the groups showed statistical significance in Group A on days $7^{\text {th }}, 15^{\text {th }}$ and $30^{\text {th }}$.

Fusobacterium. nucleatum and Capnocytophaga were the most predominant organisms isolated in both experimental and control sites. Prevotella. intermedia was isolated in lesser numbers in both experimental and control sites. [Figure: 7,8]. Prophyromonas. gingivalis was not isolated in any of the site.

A lesser number of patients with Prevotella. intermedia was found in this study. A mean decrease in Fusobacterium. Nucleatum, Capnocytophaga, Prevotella. intermedia were observed in both the groups when compared from day 0 to day 30 . Comparison between the groups showed statistically high significance in Group A on days 15 and 30, wherein there was a significant reduction in the bacterial count in the Experimental Group when compared to that of the Control Group after the application of neem extract gel.

\section{DISCUSSION}

Treatment of periodontal disease is routinely based on oral hygiene procedures and root debridement which reduces the periodontal bacteria. ${ }^{15}$ These bacteria are one of the significant factors in the etiology of periodontal disease. ${ }^{16,17}$ Mechanical debridement is effective in both disturbing the biofilm and reducing the bacterial load. However, in few patients mechanical instrumentation of the affected area is not sufficient to control the disease. Failure to obtain favourable response in these patients is probably due to the presence of the microbial species with invasive potential in sulcular epithelium, subepithelial connective tissue and dentinal tubules. In few instances, periodontal pathogens may recolonize the debrided sites from reservoirs on the surfaces of the tongue and tonsils. In such conditions, incorporation of a chemotherapeutic agent in conjunction with mechanical instrumentation provides an additional antimicrobial effect offering increased opportunities of disease control. ${ }^{18}$

The systemic use of antibiotics along with mechanical debridement suppresses or eradicates pathogenic microbiota and dramatically improves the clinical parameters like bleeding on probing (BOP), probing pocket depth (PPD) and clinical attachment level (CAL). ${ }^{19}$ The systemic use of antibiotics though useful is not free from their inherent adverse effect and disadvantages. Prominent among them are unpredictable drug concentrations at the base of the pocket, questionable substantivity and risk of developing resistant microbial population at non-oral body sites. ${ }^{20}$

To overcome these undesirable effects several local drug delivery system and devices have been developed in the past decade. The concept that the local delivery of antibiotic into the periodontal pocket achieves greater concentrations of the drug than available with systemic delivery is very promising and appealing. It is considered that higher drug concentration will prove bacteriostatic at minimum inhibitory concentration available with systemic administration. Equally important is the fact local delivery of antibiotics exhibits negligible impact on the microflora residing in the other regions of the body.

Periodontal pocket is a unique site of infection. It is readily accessible to the clinician and can be individually treated and monitored to determine the treatment response. It also provides an opportunity to treat only those sites that are deemed to be in need of treatment by mechanical debridement and local administration of antimicrobial agents. The various local drug delivery system available are pocket irrigants, sustained release devices and controlled release devices and the agents which are commonly used are tetracycline, ${ }^{21}$ metronidazole, ${ }^{22}$ doxycycline, ${ }^{23}$ Chlorhexidine, ${ }^{24}$ and minocycline. ${ }^{25}$ 
The successful long-term management of adult periodontitis requires a proper maintenance of the results obtained after treatment i.e. the reduced levels of periodontopathic bacteria. This can be successfully done with the adjunctive use of antimicrobials. ${ }^{26}$

Recent studies of traditional medicine has revealed the use of neem in treating oral infections. It has been used from time memorial as the preferred tool for maintaining healthy gums and teeth. Neem has multivarious actions like antibacterial, astringent, antiseptic, anti-inflammatory, antiviral and antimicrobial properties. It is easily available and has no known adverse reactions. Hence it has been used as a local delivery gel in this study in the treatment of patients with chronic periodontitis.

\section{TABLES}

Table: 1 Plaque Index Descriptive Statistics

\begin{tabular}{|c|c|c|c|c|c|}
\hline Duration & Group & N & Mean & Std. Deviation & Std Error Mean \\
\hline \multirow{2}{*}{ Baseline } & $\mathbf{A}$ & 20 & 2.8875 & .1716 & 3.837E-02 \\
\hline & B & 20 & 2.7875 & .2470 & $5.524 \mathrm{E}-02$ \\
\hline \multirow[b]{2}{*}{ One month } & $\mathbf{A}$ & 20 & 1.1875 & .5434 & .1215 \\
\hline & B & 20 & 1.8375 & .3996 & $8.936 \mathrm{E}-02$ \\
\hline \multirow{2}{*}{ Three months } & $\mathbf{A}$ & 20 & .8750 & .2500 & 5.59.E-02 \\
\hline & B & 20 & 1.3375 & .4749 & .1062 \\
\hline \multirow{2}{*}{ Six months } & $\mathbf{A}$ & 20 & .9000 & .2487 & 5.561E-.02 \\
\hline & B & 20 & 1.3500 & .4617 & .1032 \\
\hline
\end{tabular}

Table: 2 Gingival Index-Descriptive Statistics

\begin{tabular}{|c|c|c|c|c|}
\hline Duration & Group & Mean & Std. Deviation & $\mathbf{N}$ \\
\hline \multirow[t]{3}{*}{ Baseline } & $\mathbf{A}$ & 2.8000 & .2236 & 20 \\
\hline & B & 2.8500 & .2206 & 20 \\
\hline & Total & 2.8250 & .2207 & 40 \\
\hline \multirow[t]{3}{*}{ One month } & $\mathbf{A}$ & 1.3250 & .4595 & 20 \\
\hline & B & 1.6750 & .4667 & 20 \\
\hline & Total & 1.5000 & .4903 & 40 \\
\hline \multirow[t]{3}{*}{ Three months } & $\mathbf{A}$ & 0.8250 & .2447 & 20 \\
\hline & B & 1.4125 & .2724 & 20 \\
\hline & Total & 1.1188 & .3922 & 40 \\
\hline \multirow[t]{3}{*}{ Six months } & $\mathbf{A}$ & 0.7375 & .2361 & 20 \\
\hline & B & 1.3250 & .2308 & 20 \\
\hline & Total & 1.0313 & .3763 & 40 \\
\hline
\end{tabular}

Table: 3a Probing Pocket Depth

\begin{tabular}{|lllll|}
\hline Pair & Probing Pocket Depth & t & df & Sig. (2-tailed) \\
\hline Pair 1 & Baseline- One month & $\mathbf{1 8 . 3 8 0}$ & $\mathbf{1 9}$ & $\mathbf{. 0 0 0}$ \\
\hline Pair 2 & Baseline-Three months & $\mathbf{1 3 . 6 5 3}$ & $\mathbf{1 9}$ & $\mathbf{. 0 0 0}$ \\
\hline Pair 3 & Baseline- Six months & $\mathbf{1 7 . 4 9 0}$ & $\mathbf{1 9}$ & $\mathbf{. 0 0 0}$ \\
\hline Pair 4 & One month-Three months & $\mathbf{2 . 6 9 8}$ & $\mathbf{1 9}$ & $\mathbf{. 0 1 4}$ \\
\hline Pair 5 & One month-Six months & $\mathbf{5 . 1 4 6}$ & $\mathbf{1 9}$ & $\mathbf{. 0 0 0}$ \\
\hline \hline Pair 6 & Three months-Six months & $\mathbf{3 . 1 9 9}$ & $\mathbf{1 9}$ & $\mathbf{. 0 0 5}$ \\
\hline
\end{tabular}


Table: 3b Clinical Attachment Level

\begin{tabular}{|lllll|}
\hline Pair & Clinical Attachment Level & t & df & Sig. (2-tailed) \\
\hline Pair 1 & Baseline- One month & $\mathbf{1 0 . 5 1 4}$ & $\mathbf{1 9}$ & $\mathbf{. 0 0 0}$ \\
\hline Pair 2 & Baseline-Three months & $\mathbf{9 . 9 7 0}$ & $\mathbf{1 9}$ & $\mathbf{. 0 0 0}$ \\
\hline Pair 3 & Baseline- Six months & $\mathbf{1 2 . 8 5 6}$ & $\mathbf{1 9}$ & $\mathbf{. 0 0 0}$ \\
\hline Pair 4 & One month-Three months & $\mathbf{2 . 3 4 9}$ & $\mathbf{1 9}$ & $\mathbf{. 0 3 0}$ \\
\hline Pair 5 & One month-Six months & $\mathbf{7 . 0 2 5}$ & $\mathbf{1 9}$ & $\mathbf{. 0 0 0}$ \\
\hline Pair 6 & Three months-Six months & $\mathbf{4 . 3 3 3}$ & $\mathbf{1 9}$ & $\mathbf{. 0 0 0}$ \\
\hline
\end{tabular}

\section{SUMMARY AND CONCLUSION}

The aim of this study was to explore the efficacy of local delivery of Azadirachta indica (neem) extract gel and evaluate the periodontal tissue response by recording clinical parameters like Plaque index, Gingival index, Probing Pocket Depth, Clinical Attachment Level and Micro biological Evaluation.

Neem leaf extract was incorporated into a biodegraded polymer (Carbopol and Gellan) and formulated in a gel form. The dosage used was $20 \mathrm{mg} / \mathrm{gm}$ to be delivered at the target site. The drug was formulated in the gel form for the ease of placement and retention at the target site following placement.

A total of 40 sites were selected from 20 patients with chronic periodontitis in the age group of 30 to 55 years of both sexes, with pocket depth of $\leq 5 \mathrm{~mm}$ with radiographic evidence of bone loss bilaterally. A splitmouth design was employed and all the clinical parameters were recorded at baseline, one month, three months and at six months, at the target groups. However, the microbiological evaluation was carried out for 20 sites in 10 patients and the microbial counts were recorded at baseline, 7 days, 15 days and 30 days post operatively. A limited sample size of 20 patients in the present study is attributed to the following reasons, namely-Selection of two identical defects contra-laterally in the same arch is rather difficult and patients may not agree to come for a longer follow up of more than six months.

The problems of clinical periodontal probing were minimized by using a constant pressure probe (Brock probe ${ }^{\mathrm{TM}}$ ) and customized acrylic stents with guiding grooves for reproducing probing sites and directions. From a clinical point of view, neem gel was very well tolerated with no overt side effects. The post treatment phase was uneventful.Within limits superimposed by a relatively smaller sample size, the present study showed improvements in clinical as well as microbiological parameters both at the control (Placebo) and experimental (neem) sites. However, the experimental site showed better results compared to the control.

From the results obtained the following conclusions were arrived-the neem gel introduced in the study consists of delivering sufficient quantity of the drug to provide adequate therapeutic levels at the target site as a beneficial treatment modality, Local delivery with the use of neem extract gel is a simple and non invasive technique with no reported adverse effects and it reduces the percentage of microorganisms in periodontal pockets and improvements in clinical parameters was evident. As evident from this study, it can be assumed that neem extract gel therapy along with scaling and root planing is more effective than scaling and root planing alone and may form the future for potential application in periodontal therapy.

\section{REFERENCES}

[1]. Chirugien Dentiste Le, Traite des ou. Dents. 2vols. Paris, Mariette J. 1728. Reprinted in facsimile, Paris, Prelat, 1961. (An English translation by Lilian Lindsay appeared in 1946, published by Buttersworth and Company, London). Cobb CM. Non surgical pocket therapy: Pharmacotherapeutics. Ann Periodontol 1996; 1: 491-566.

Carranza FA, Newman MG. Clinical periodontology. $9^{\text {th }}$ ed. 2003; Harcourt (Pvt.) Ltd., India.

Rams TE and Slots J. Local delivery of antimicrobial agents in the periodontal pocket. Periodontol 2000. 1996; 10:139-159. Journal of Ethno Pharmacology. 2004; 90: 99-103.

Zee KY et al. Bacterial morphotypes of three day old plaque in Chinese.

J Clin Periodontol 1996; 23: 403-406.

Genco. Antibiotics in the treatment of periodontal disease. J Periodontol 1981; 52: 545-558.

Moore WEC. Microbiology of periodontal disease. J Periodontol Res 1987; 22: 335-341.

[10]. Sanz et al. Differences in composition of subgingival microbiota of two periodontitis populations of different geographical origin - A comparison between Spain and Netherlands. Eur J Oral Sci 2000; 108: 383-392.

[11]. Bergenholtz A, Al-Harbi N, Al-Hummayani FM,Anton P, Al-Kahtani S. The accuracy of the Vivacare true pressure sensitive periodontal probe system in terms of probing force.J Clin Periodontol 2000; 27: 93-98.

[12]. Meadows CL, Gher ME, Quintero G, Lafferty TA. A comparison of Polylactic acid granules and decalcified Freeze-dried bone allograft in human periodontal osseous defects. J Periodontol 1993; 64:103-109.

[13]. Esposita E, Carotta V. Int J Pharm 1996; 142: 9-23.

[14]. Goodson J, Cuginim, Kent RL et al. Multicentered evaluation of tetracycline fibre therapy to clinical response. J Perio Res 1991 ; 26: 371 .

[15]. Ianomo J, Lie P, Ellingsen $\mathrm{BH}$ et al. Clinical responses to subgingival application of metronidazole $25 \%$ gel compared to the effect of sub-gingival scaling in adult periodontitis. J Clin Periodontol 1992; 19: Part II, 723.

[16]. Garrette S, Johnson L, Drisko VT et al. Three multicentered studies evaluating locally delivered Doxycycline hyaclate, placebo control, oral hygiene and scaling and root planning in the treatment of periodontitis. J Periodontol 1999; 70: $490-503$. 
[17]. Braswel L, Offenbacker S, Fritz N, Vandy KE. Local delivery of minocycline to periodontal lesion in a slow release polymer. J Dent Res 1992; 71: Abst 1115.

[18]. Okuda K, Wolft T, Oliver R, Osborn J, Stoltenberg L, Berueter J, Anderson L. Minocycline slow release formulation effect on subgingival bacteria. J Periodontol 1992; 63: 73-79.

[19]. Cobb CM. Non surgical pocket therapy-mechanical. Ann Periodontol 1996; 1:443-490.

[20]. Roxanne A, Greenstein LG. Clinical and microbioligical response to nonsurgical mechanical periodontal therapy. Periodontology 2000. 1995; 9: 14-22.

[21]. Killoy, Polson. Controlled local delivery of antimicrobials in treating periodontitis. DCNA. 270-273.

[22]. Mandel ID. Chemotherapeutic agents for controlling plaque and Gingivitis. J Clin Periodontol 1988; 15: 488-498.

[23]. Mousques T, Listgarten MA, Philips WR. Effect of scaling and root planing on subgingival microflora. J Periodontol Res 1980; 15: 144-151.

[24]. Hunter FM, Martin MD, Stevenson ARL. Evaluation of a pressure sensitive periodontal probe tip my measuring its histologic relation to periodontal attachments. J Perio Res 1992; 71: 581.

[25]. Quintero G, Mellanig JT, Gambill VM. A six month clinical evaluation of Demineralized Freeze Dried Bone Allograft in periodontal osseous defects. J Periodontol 1982; 53: 726-730.

[26]. Oreamuno S, Lekovei V, Kenney EB, Carranza FA, Takei HA, Prokic B.Comperative clinical study porous Hydroxappitite and DFDBA in human periodontal defects. J Periodontol 1990; 61: 399-405.

[27]. Anantanarayan R, Paniker CKJ. Morphology and Physiology of bacteria. Chapter 2, Textbook of Microbiology. $3^{\text {rd }}$ ed: 7-23.

\section{LEGENDS FOR PHOTOGRAPHS:}

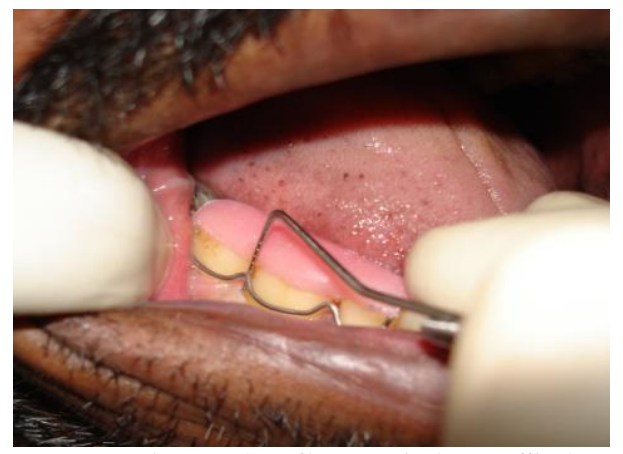

Figure 1.a Group A (Test Site) Clinical Parameters and Probing Pocket depth measured at Baseline

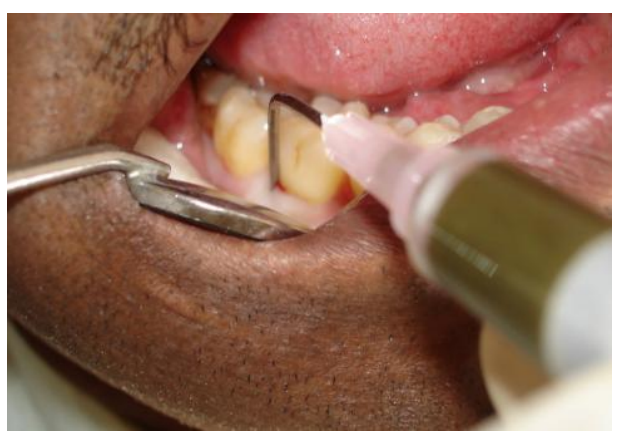

Figure 2.a -at Site A (Test Site) Placement of Gel 1 (Neem extract Gel)

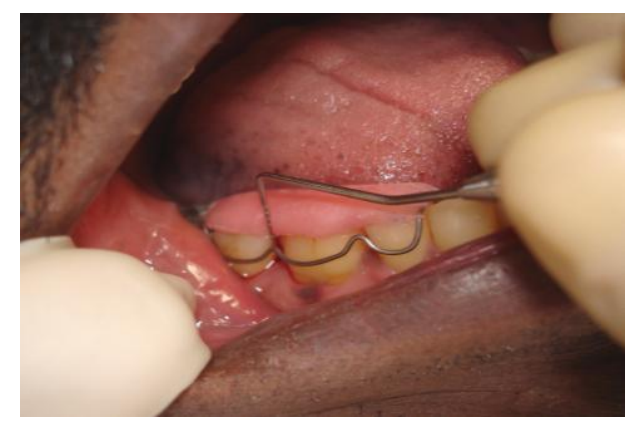

Figure 3.a Group A (Test Site)

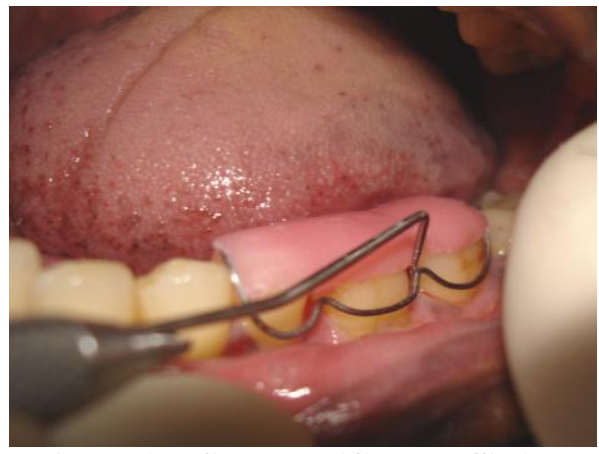

Figure 1.b Group B (Control Site)

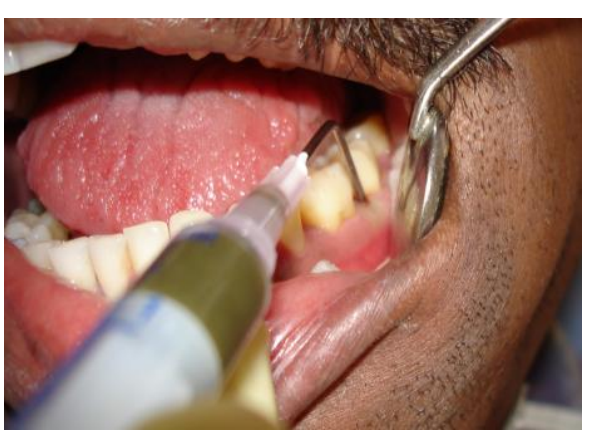

Figure 2.b - at Site B (Control Site) Placement of Gel 2 (Placebo Gel)



Figure 3.b Group B (Control Site)

Clinical Parameters and Probing Pocket depth measured at one month 


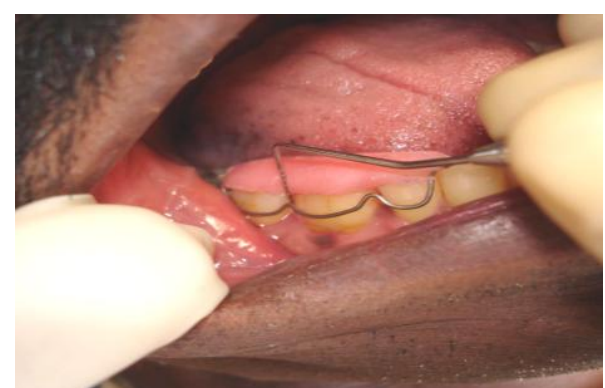

Figure 4.a Group A (Test Site)

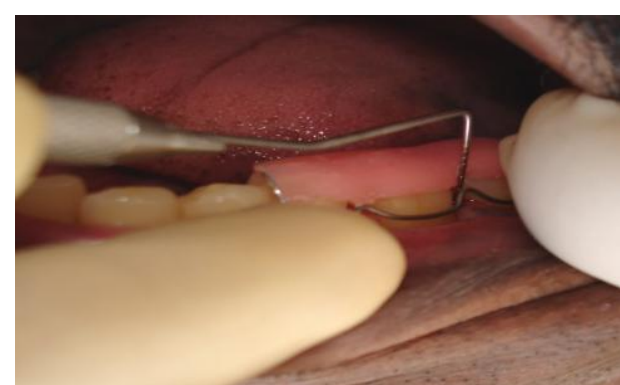

Figure 4.b Group B (Control Site)

Clinical Parameters and Probing Pocket depth measured at six months

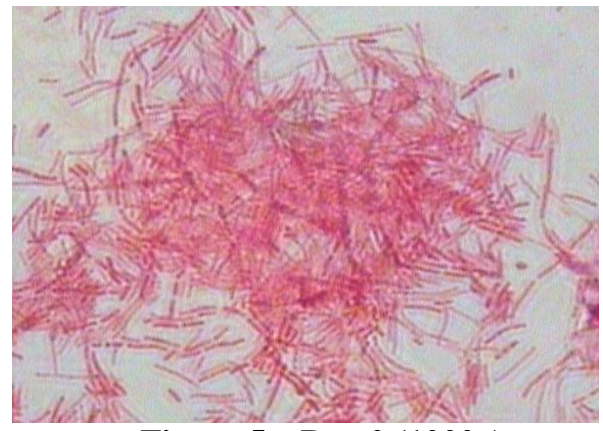

Figure 5 - Day 0 (1000x)



Figure 6 - Day 30 (1000x)

Photomicrograph of Gram's stain

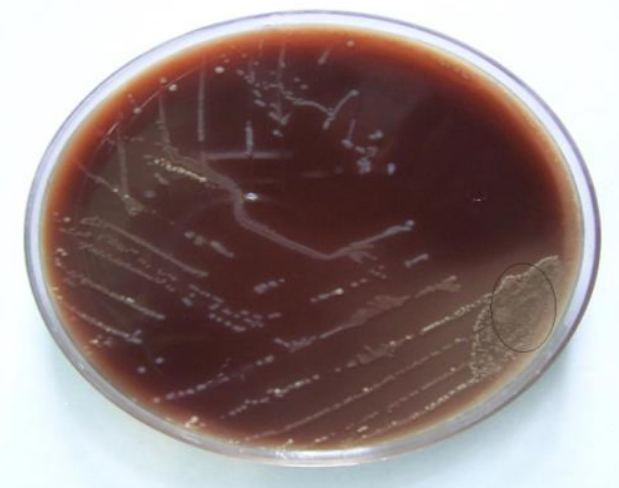

Figure 7

Figure 7 - Culture plate with spreading grey colonies (suggestive of Capnocytophaga ) and grey nacreous colonies ( suggestive of F. nucleatum)

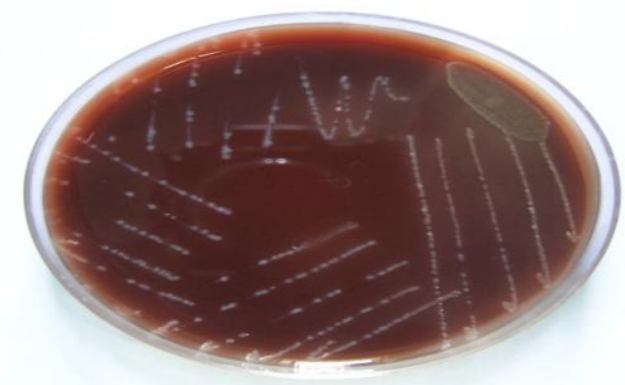

Figure 8

Figure 8- Culture plate with black pigmented colonies (suggestive of $P$. intermedia) and grey nacreous colonies (suggestive of $F$. nucleatum) 